\section{Military Technical College Kobry El-Kobbah, Cairo, Egypt}

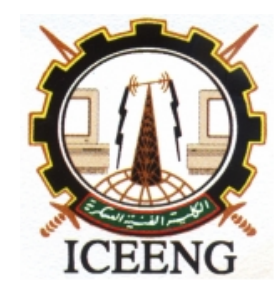

\title{
Circular pipe flow of a dusty Casson fluid considering the hall effect
}

$$
\text { By }
$$

Hazem Ali Attia * Karem Mahmoud Ewis ** Ibrahim Hamdy Abd Elmaksoud***

\section{$\underline{\text { Abstract: }}$}

In the present paper, the time varying flow of a dusty viscous incompressible conducting non-Newtonian Casson fluid through a circular pipe is studied considering the Hall effect. A constant pressure gradient in the axial direction and a external uniform magnetic field perpendicular to the flow direction are applied. A numerical solution is obtained for the governing nonlinear equations using finite differences and the effect of the different physical parameters on the velocity distributions of fluid and dust particles are reported.

\section{Keywords:}

Magneto-fluid mechanics, computational methods, flow in channels, circular pipe flow, non-Newtonian fluid, Casson fluid, Hall current.

* Faculty of Engineering, Fayoum University, Fayoum, Egypt

** Faculty of Engineering, Fayoum University, Fayoum, Egypt

*** Faculty of Engineering, Fayoum University, Fayoum, Egypt 


\section{Introduction:}

The flow of a dusty conducting fluid inside an infinite circular pipe in the presence of a transverse magnetic field has many applications such as magnetohydrodynamic (MHD) generators, pumps, accelerators, and flowmeters etc. The performance and efficiency of these devices are affected by the existence of solid particles in the form of ash or soot as a result of the corrosion and wear activities and/or the combustion processes in MHD generators and plasma MHD accelerators. If the particle concentration becomes high, mutual particle interaction leads to higher particle-phase viscous stresses and can be accounted for by endowing the particle phase by the so-called particle-phase viscosity. Many studies deal with theoretical modeling and experimental measurements of the particle-phase viscosity in a dusty fluid (Soo 1969, Gidaspow et al. 1986, Grace 1982, and Sinclair et al. 1989).

The flow of an electrically conducting fluid in a circular pipe has been investigated by many resaerchers (Gadiraju et al. 1992, Dube et al. 1975, Ritter et al. 1977, and Chamkha 1994). Gadiraju et al. (1992) studied the steady two-phase vertical flow in a pipe. Dube et al. (1975) and Ritter et al. (1977) investigated solutions for unsteady dusty-gas flow in a circular pipe in the absence of a magnetic field and particle-phase viscous stresses. Chamkha (1994) obtained exact solutions which generalize the results reported in Dube et al. 1975 and Ritter et al. 1977 by the inclusion of the magnetic and particle-phase viscous effects where the Hall effect is neglected.

A number of practically important fluids in industry such as molten plastics, polymers, pulps and foods have non-Newtonian fluid behavior (Nakayama et al. 1988). Due to the growing use of these non-Newtonian materials, in various manufacturing and processing industries, efforts are directed to understand their flow characteristics. Many of the inelastic non-Newtonian fluids, encountered in chemical engineering processes, are known to follow the so-called "power-law model" in which the shear stress varies according to a power function of the strain rate (Metzner et al. 1965).

In the present paper, the unsteady flow of a dusty viscous incompressible electrically conducting non-Newtonian Casson fluid through a circular pipe is investigated considering the Hall effect. The particle phase is assumed to be incompressible pressureless and electrically non-conducting. The flow in the pipe starts impulsively from rest through the application of a constant axial pressure gradient. The governing nonlinear momentum equations for both the fluid and particle-phases are solved numerically using finite differences. The effect of the Hall current, the nonNewtonian fluid characteristics and the particle-phase viscosity on the velocity of the fluid and particle-phases are reported and discussed. 


\section{Governing Equations:}

Consider the time varying and axisymmetric horizontal flow of a dusty electrically conducting non-Newtonian Casson fluid through an infinitely long circular pipe of radius $d$ driven by a constant pressure gradient in the axial direction. A uniform magnetic field is applied perpendicular to the flow direction. The Hall current is taken into consideration but the magnetic Reynolds number is assumed to be very small, consequently the induced magnetic field is neglected (Sutton et al. 1965). We assume that both phases behave as viscous fluids and that the volume fraction of suspended particles is finite and constant (Chamkha 1994). Therefore the governing momentum equations are given as

$\rho \frac{\partial V}{\partial t}=-\frac{\partial P}{\partial z}+\frac{1}{r} \frac{\partial}{\partial r}\left(\mu r \frac{\partial V}{\partial r}\right)+\frac{\rho_{p} \phi}{1-\phi} N\left(V_{p}-V\right)-\frac{\sigma B_{o}^{2} V}{1+m^{2}}$

$\rho_{p} \frac{\partial V_{p}}{\partial t}=\frac{1}{r} \frac{\partial}{\partial r}\left(\mu_{p} r \frac{\partial V_{p}}{\partial r}\right)+\rho_{p} N\left(V-V_{p}\right)$

where $t$ is the time, $r$ is the distance in the radial direction, $V$ is the fluid-phase velocity, $V_{p}$ is the particle-phase velocity, $\rho$ is the fluid-phase density, $\rho_{p}$ is the particle-phase density, $\partial P / \partial \mathrm{z}$ is the fluid pressure gradient, $\varphi$ is the particle-phase volume fraction, $N$ is a momentum transfer coefficient (Chamkha 1994), $\sigma$ is the fluid electrical conductivity, $m=\sigma \beta B_{o}$ is the Hall parameter, $\beta$ is the Hall factor (Sutton et al. 1965), $B_{o}$ is the magnetic induction, $p$ is the particle-phase viscosity which is assumed constant, and is the apparent viscosity of the fluid which is given by,

$$
\mu=\left(K_{c}+\sqrt{\frac{\tau_{o}}{|\partial V / \partial r|}}\right)^{2}
$$

where $K_{c}$ is the coefficient of viscosity of a Casson fluid, $\tau_{\mathrm{o}}$ is the yield stress, and $|\partial V / \partial r|$ is the magnitude of the velocity gradient which is always positive regardless of the sign of $\partial V / \partial r$. In this work, $\rho, \rho_{p}, p, \varphi$, and $B_{o}$ are all constant.

The initial and boundary conditions of the problem are given as

$$
\begin{aligned}
& V(r, 0)=0, V_{p}(r, 0)=0, \\
& \frac{\partial V(0, t)}{\partial r}=0, \frac{\partial V_{p}(0, t)}{\partial r}=0, V(d, t)=0, V_{p}(d, t)=0
\end{aligned}
$$


Introducing the following dimensionless variables and parameters

$\bar{r}=\frac{r}{d}, \bar{t}=\frac{t K_{c}}{\rho d^{2}}, G_{o}=-\frac{\partial P}{\partial z}, k=\frac{\rho_{p} \phi}{\rho(1-\phi)}, \bar{\mu}=\frac{\mu}{K_{c}}, \bar{V}(r, t)=\frac{K_{c} V(r, t)}{G_{o} d^{2}}, \bar{V}_{p}(r, t)=\frac{K_{c} V_{p}(r, t)}{G_{o} d^{2}}, \alpha=N d^{2} \rho / K_{c}$ is the inverse Stoke's number,

$\beta=\mu_{p} / K_{c}$ is the viscosity ratio,

$\tau_{D}=\tau_{o} / G_{o} d$ is the Casson number (dimensionless yield stress),

$H_{a}=B_{o} d \sqrt{\sigma / K_{c}}$ is the Hartmann number (Sutton et al. 1965).

Equations (1)-(3) can be written as (the bars are dropped for simplicity)

$\frac{\partial V}{\partial t}=1+\sqrt{\mu} \frac{\partial^{2} V}{\partial r^{2}}+\frac{\mu}{r} \frac{\partial V}{\partial r}+k \alpha\left(V_{p}-V\right)-\frac{H_{a}^{2} V}{1+m^{2}}$

$\frac{\partial V_{p}}{\partial t}=\beta\left(\frac{\partial^{2} V_{p}}{\partial r^{2}}+\frac{1}{r} \frac{\partial V_{p}}{\partial r}\right)+\alpha\left(V-V_{p}\right)$

$\mu=\left(1+\sqrt{\frac{\tau_{D}}{|\partial V / \partial r|}}\right)^{2}$

$V(r, 0)=0, V_{p}(r, 0)=0$,

$\frac{\partial V(0, t)}{\partial r}=0, \frac{\partial V_{p}(0, t)}{\partial r}=0, V(1, t)=0, V_{p}(1, t)=0$

The volumetric flow rates and skin-friction coefficients for both the fluid and particle phases are defined, respectively, as (Chamkha 1994)

$Q=2 \pi \int_{0}^{1} r V(r, t) d r, Q_{p}=2 \pi \int_{0}^{1} r V_{p}(r, t) d r, C=-\frac{\partial V(1, t)}{\partial r}, C_{p}=-\beta k \frac{\partial V_{p}(1, t)}{\partial r}$

\section{Results and Discussion:}

Equations (4) and (5) represent a coupled set of nonlinear partial differential equations which are solved numerically under the initial and boundary conditions (6), using the finite difference method. A linearization technique is first applied to replace the nonlinear terms at a linear stage, with the corrections incorporated in subsequent iterative steps until convergence is reached. Then the Crank-Nicolson implicit method (Evans et al. 2000) is used at two successive time levels. An iterative scheme is used to solve the linearized system of difference equations where the solution at a certain time 
step is chosen as an initial guess for next time step and the iterations are continued till convergence, within a prescribed accuracy. Finally, the resulting block tridiagonal system is solved using the generalized Thomas algorithm (Evans et al. 2000). Computations are carried out for $\alpha=1$ and $k=10$. Grid-independence studies show that the computational domain $0<t<\infty$ and $0<r<1$ can be divided into intervals with step sizes $t=0.0001$ and $r=0.005$ for time and space respectively.

Figures 1-4 present the variation with time of the profiles of the velocity of the fluid $V$ and dust particles $V_{p}$, respectively for different values of the Bingham number $\tau_{\mathrm{D}}$ and the Hall parameter $m$ and for $H a=0.5$ and $\beta=0.5$. Both $V$ and $V_{p}$ increase with time but $V$ reaches the steady-state faster than $V_{p}$ for all values of $\tau_{D}$. It is clear from the Figs. that increasing $\tau_{D}$ increases both $V$ and $V_{p}$ while its effect on their steady-state times can be ignored. It is clear from the figures that increasing $m$ increases $V$ and, in turn, $V_{p}$ due to the decrease in the effective conductivity $\left(\sigma /\left(1+m^{2}\right)\right)$ which reduces the damping magnetic force on $V$. It is shown that the influence of the Hall parameter $m$ on $V$ is more apparent for higher values of $\tau_{D}$.

Table 1 presents the steady state values of the fluid-phase volumetric flow rate $Q$, the particle-phase volumetric flow rate $Q_{p}$, the fluid-phase skin friction coefficient $C$, and the particle-phase skin friction coefficient $C_{p}$ for various values of the parameters $\tau_{D}$ and $m$ and for $H a=0.5$ and $\beta=0.5$. It is clear that increasing the parameter $m$ increases $Q, Q_{p}, C$, and $C_{p}$ for all values of $\tau_{D}$. It is also shown that increasing $\tau_{D}$ increases $Q, Q_{p}$, $C$, and $C_{p}$ for all values of $m$.

Table 2 presents the steady state values of the fluid-phase volumetric flow rate $Q$, the particle-phase volumetric flow rate $Q_{p}$, the fluid-phase skin friction coefficient $C$, and the particle-phase skin friction coefficient $C_{p}$ for different values of the parameters $m$ and $\beta$ and for $H a=0.5$ and $\tau_{D}=0$. It is clear that, increasing $m$ increases $Q, Q_{p}, C$, and $C_{p}$ for all values of $\beta$ and its effect becomes more apparent for smaller values of $\beta$. Increasing the parameter $\beta$ decreases the quantities $Q, Q_{p}$, and $C$, but increases $C_{p}$ for all values of $m$. 


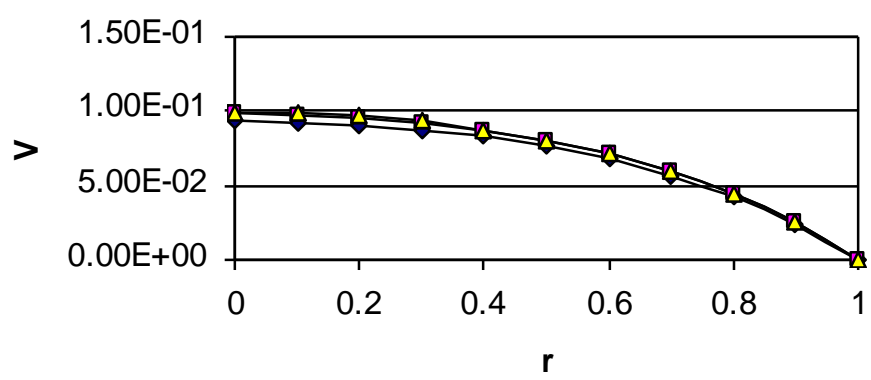

$\neg \mathrm{t}=0.5 \rightarrow \mathrm{t}=1 \rightarrow \mathrm{t}=2$

(a) $\tau_{\mathrm{D}}=0.0$

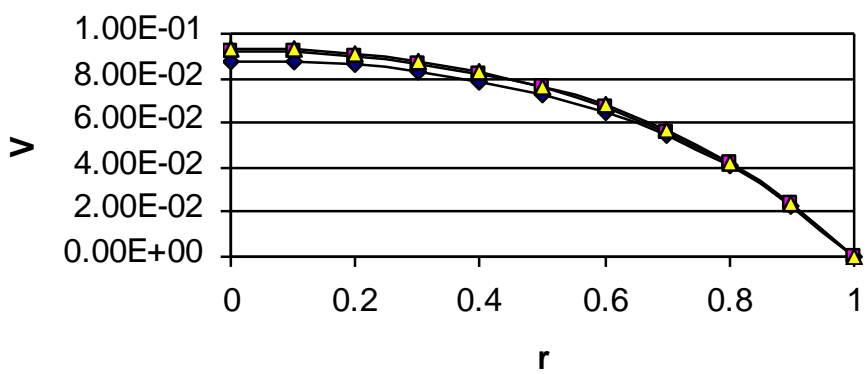

$\multimap \mathrm{t}=0.5 \multimap \mathrm{t}=1 \multimap \mathrm{t}=2$

(b) $\tau_{\mathrm{D}}=0.025$

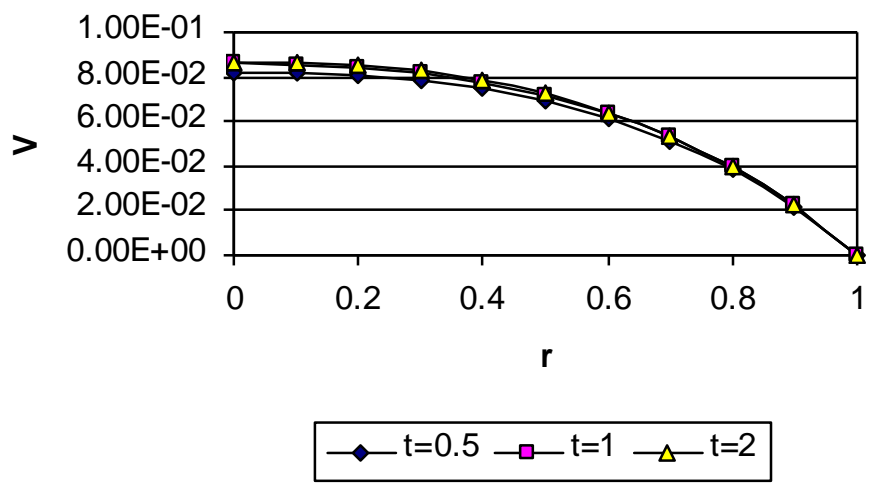

(c) $\tau_{\mathrm{D}}=0.05$

Figure (1): Time development of the profile of $V$ for various values of $\tau_{D}\left(m=0, H_{a}=0.5\right.$, $\beta=0.5)$ 


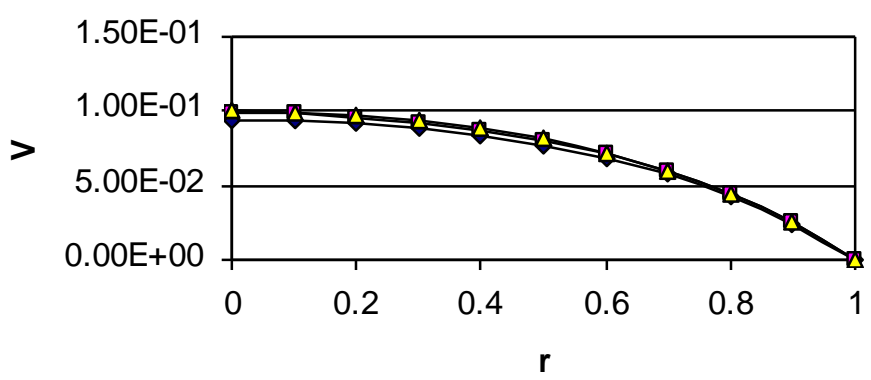

$\leadsto \mathrm{t}=0.5 \rightarrow \mathrm{t}=1 \multimap \mathrm{t}=2$

(a) $\tau_{\mathrm{D}}=0.0$

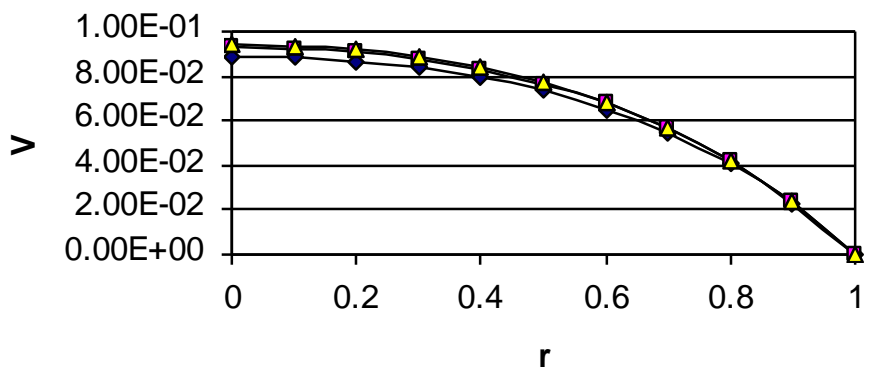

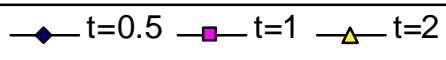

(b) $\tau_{\mathrm{D}}=0.025$

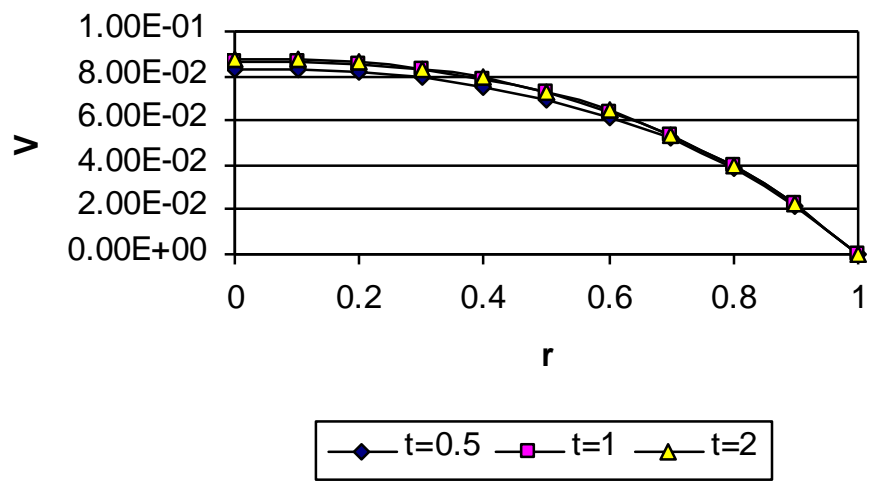

(c) $\tau_{\mathrm{D}}=0.05$

Figure (2): Time development of the profile of $V$ for various values of $\tau_{D}\left(m=1, H_{a}=0.5\right.$, $\beta=0.5)$ 


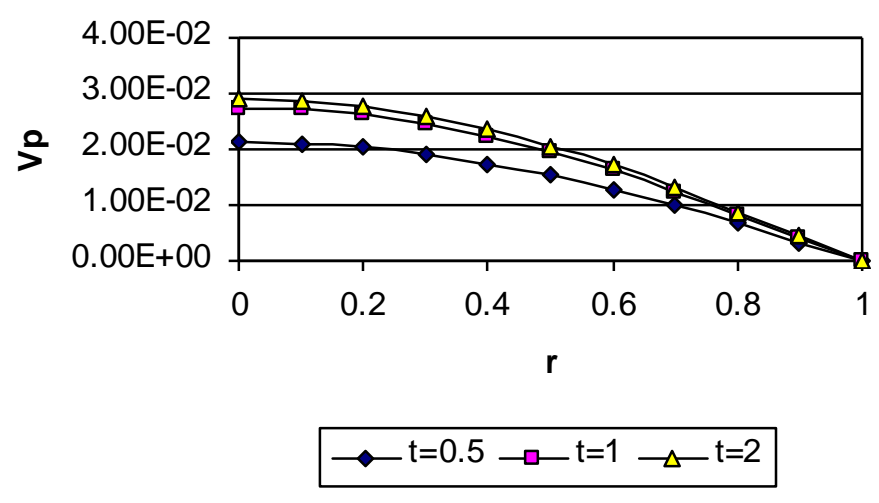

(a) $\tau_{\mathrm{D}}=0.0$

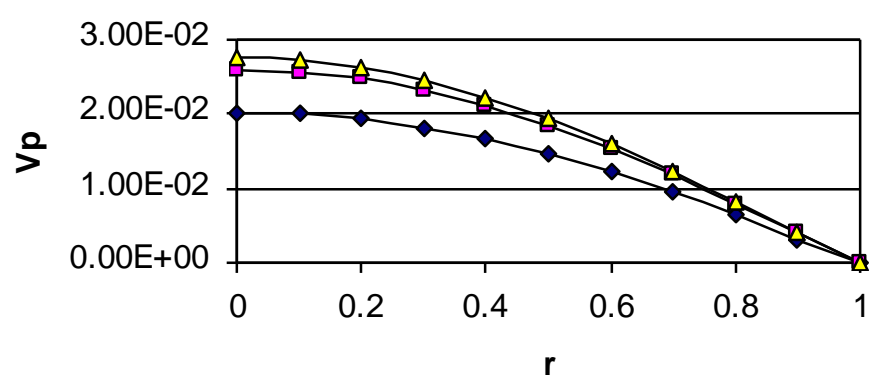

$\prec \mathrm{t}=0.5 \multimap \mathrm{t}=1 \multimap \Delta \mathrm{t}=2$

(b) $\tau_{\mathrm{D}}=0.025$

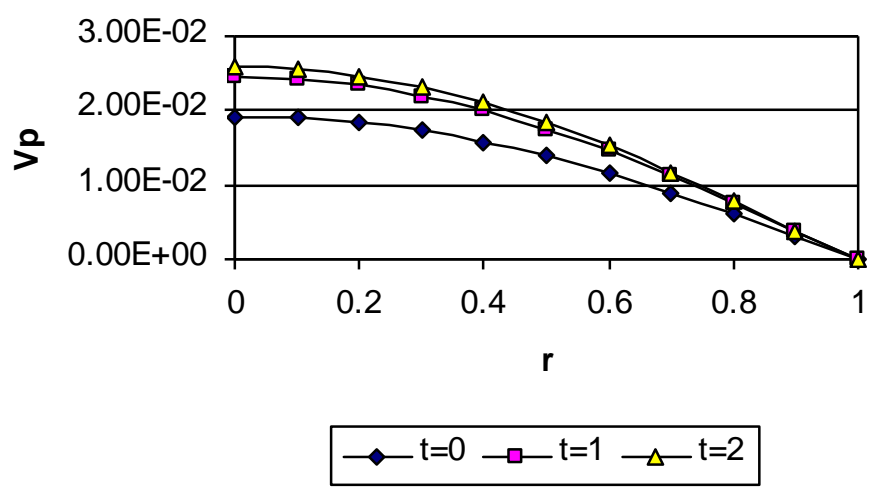

(c) $\tau_{\mathrm{D}}=0.05$

Figure (3): Time development of the profile of $V_{p}$ for various values of $\tau_{D}(m=0$, $\left.\mathrm{H}_{\mathrm{a}}=0.5, \beta=0.5\right)$ 


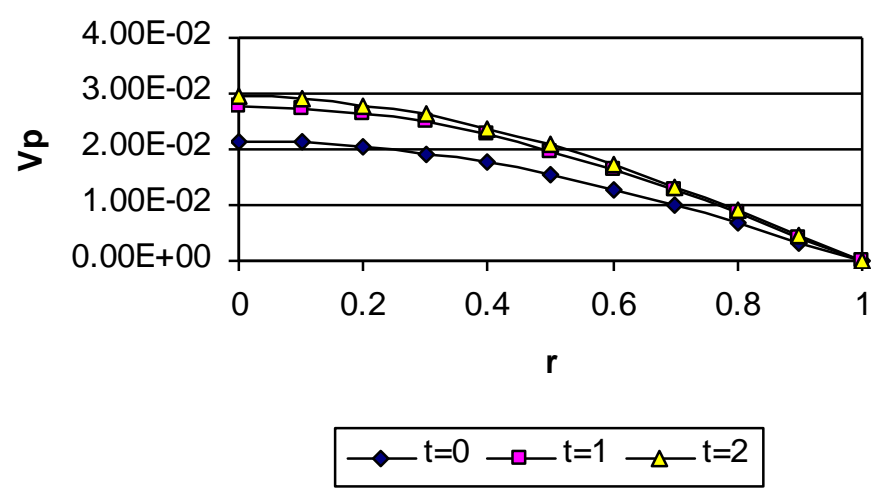

(a) $\tau_{\mathrm{D}}=0.0$

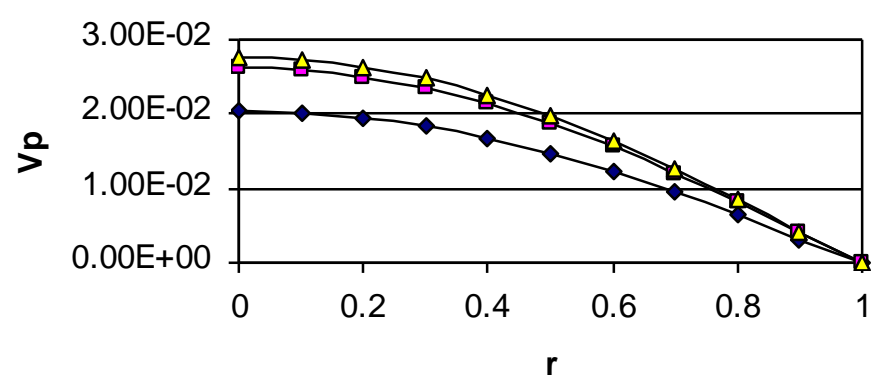

$\multimap \mathrm{t}=0 \multimap \mathrm{t}=1 \multimap \mathrm{t}=2$

(b) $\tau_{\mathrm{D}}=0.025$

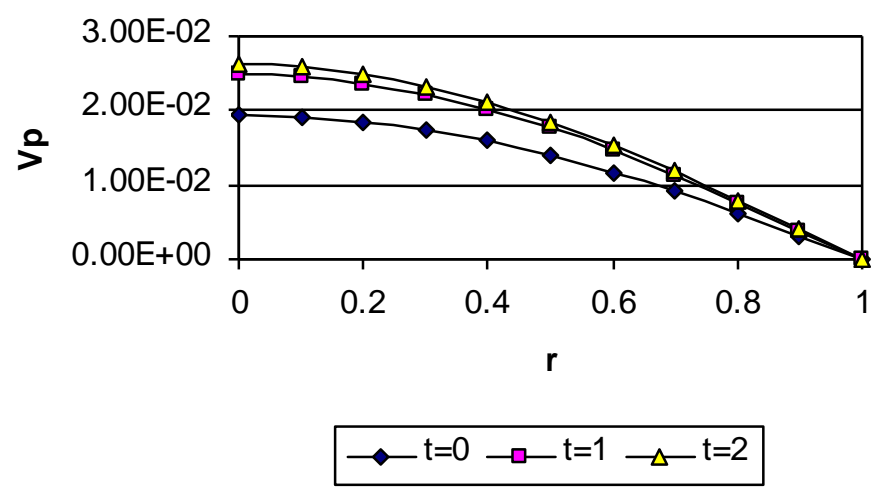

(c) $\tau_{\mathrm{D}}=0.05$

Figure (4): Time development of the profile of $V_{p}$ for various values of $\tau_{D}(m=1$, $\mathrm{H}_{\mathrm{a}}=0.5, \beta=0.5$ ) 
Table (1): The steady state values of $\mathrm{Q}, \mathrm{Q}_{\mathrm{p}}, \mathrm{C}, \mathrm{C}_{\mathrm{p}}$ for various values of $\mathrm{m}$ and $\tau_{\mathrm{D}}$

\begin{tabular}{|c|c|c|c|}
\hline $\boldsymbol{\tau}_{\mathbf{D}}=\mathbf{0}$ & $\mathrm{m}=0$ & $\mathbf{m = 1}$ & $\mathbf{m = 2}$ \\
\hline $\mathbf{Q}$ & 0.1763 & 0.1779 & 0.1789 \\
\hline $\mathbf{Q}_{\mathbf{p}}$ & 0.0425 & 0.0429 & 0.0432 \\
\hline $\mathbf{C}$ & 0.2817 & 0.2833 & 0.2843 \\
\hline $\mathbf{C}_{\mathbf{p}}$ & 0.2106 & 0.2125 & 0.2136 \\
\hline
\end{tabular}

\begin{tabular}{|c|c|c|c|}
\hline $\boldsymbol{\tau}_{\mathbf{D}}=\mathbf{0 . 0 2 5}$ & $\mathbf{m = 0}$ & $\mathbf{m}=\mathbf{1}$ & $\mathbf{m = 2}$ \\
\hline $\mathbf{Q}$ & 0.1669 & 0.1684 & 0.1693 \\
\hline $\mathbf{Q}_{\mathbf{p}}$ & 0.0403 & 0.0406 & 0.0408 \\
\hline $\mathbf{C}$ & 0.2671 & 0.2686 & 0.2695 \\
\hline $\mathbf{C}_{\mathbf{p}}$ & 0.1995 & 0.2012 & 0.2022 \\
\hline
\end{tabular}

\begin{tabular}{|c|c|c|c|}
\hline $\boldsymbol{\tau}_{\mathbf{D}}=\mathbf{0 . 0 5}$ & $\mathbf{m = 0}$ & $\mathbf{m = 1}$ & $\mathbf{m = 2}$ \\
\hline $\mathbf{Q}$ & 0.1576 & 0.1589 & 0.1597 \\
\hline $\mathbf{Q}_{\mathbf{p}}$ & 0.0379 & 0.0383 & 0.0385 \\
\hline $\mathbf{C}$ & 0.2523 & 0.2537 & 0.2546 \\
\hline $\mathbf{C}_{\mathbf{p}}$ & 0.1883 & 0.1899 & 0.1908 \\
\hline
\end{tabular}


Table (2): The steady state values of $Q, Q_{p}, C, C_{p}$ for various values of $m$ and $\beta$

\begin{tabular}{|c|c|c|c|}
\hline$\beta=0$ & $\mathrm{~m}=0$ & $\mathrm{~m}=1$ & $\mathrm{~m}=2$ \\
\hline $\mathrm{Q}$ & 0.2653 & 0.2686 & 0.2706 \\
\hline $\mathrm{Q}_{\mathrm{p}}$ & 0.1975 & 0.1997 & 0.2009 \\
\hline $\mathrm{C}$ & 0.3759 & 0.3791 & 0.3811 \\
\hline $\mathrm{C}_{\mathrm{p}}$ & 0 & 0 & 0 \\
\hline
\end{tabular}

\begin{tabular}{|c|c|c|c|}
\hline$\beta=0.5$ & $\mathrm{~m}=0$ & $\mathrm{~m}=1$ & $\mathrm{~m}=2$ \\
\hline $\mathrm{Q}$ & 0.1763 & 0.1779 & 0.1789 \\
\hline $\mathrm{Q}_{\mathrm{p}}$ & 0.0425 & 0.0429 & 0.0432 \\
\hline $\mathrm{C}$ & 0.2817 & 0.2833 & 0.2843 \\
\hline $\mathrm{C}_{\mathrm{p}}$ & 0.2106 & 0.2125 & 0.2136 \\
\hline
\end{tabular}

\begin{tabular}{|c|c|c|c|}
\hline$\beta=1$ & $\mathrm{~m}=0$ & $\mathrm{~m}=1$ & $\mathrm{~m}=2$ \\
\hline $\mathrm{Q}$ & 0.1640 & 0.1654 & 0.1662 \\
\hline $\mathrm{Q}_{\mathrm{p}}$ & 0.0226 & 0.0228 & 0.0229 \\
\hline $\mathrm{C}$ & 0.2702 & 0.2716 & 0.2724 \\
\hline $\mathrm{C}_{\mathrm{p}}$ & 0.2231 & 0.2249 & 0.2260 \\
\hline
\end{tabular}

\section{Conclusions:}

The time varying MHD flow of a particulate suspension in a conducting non-Newtonian Casson fluid through a circular pipe is studied considering the Hall effect. The governing nonlinear partial differential equations are solved numerically using finite differences. The effect of the magnetic field parameter $\mathrm{Ha}$, the Hall parameter $\mathrm{m}$, the non-Newtonian fluid characteristics (Bingham number $\tau_{\mathrm{D}}$ ), and the particle-phase viscosity $\beta$ on the distribution of the velocity, volumetric flow rates, and skin friction coefficients of both fluid and particle-phases is reported. It is seen that increasing the magnetic field decreases the fluid and particle velocities, while increasing the Hall parameter increases both velocities. Increasing the parameter $m$ increases $Q, Q_{p}, C$, and $C_{p}$ for all values of $\tau_{D}$. The effect of the Hall parameter on the quantities $Q, Q_{p}, C$, and $C_{p}$ becomes more pronounced for smaller values of $\beta$. 
Proceedings of the $\boldsymbol{8}^{\text {th }}$ ICEENG Conference, 29-31 May, 2012

\section{References:}

[1] S.L. Soo, Pipe flow of suspensions, Appl. Sci. Res., Vol. 21, P. 68-84, 1969.

[2] D. Gidaspow, Hydrodynamics of fluidization and heat transfer, super computer modeling, Appl. Mech. Rev., Vol. 39, P. 1-23, 1986.

[3] J.R. Grace, Fluidized-Bed Hydrodynamic, Handbook of Multiphase Systems, G. Hetsoroni, Ed., Ch. 8.1, McGraw-Hill, New York, 1982.

[4] J.L. Sinclair and R. Jackson, Gas-particle flow in a vertical pipe with particleparticle interactions, AICHE J., Vol. 35, P. 1473-1486, 1989.

[5] M. Gadiraju, J. Peddieson and S. Munukutla, Exact solutions for two-phase vertical pipe flow. Mechanics Research Communications, Vol. 19, No. 1, P. 7 13, 1992.

[6] S.N. Dube and C.L. Sharma, A note on unsteady flow of a dusty viscous liquid in a circular pipe, J. Phys. Soc. Japan, Vol. 38, No.1, P. 298-310, 1975.

[7] J.M. Ritter and J. Peddieson, Transient two-phase flows in channels and circular pipes, Proc. 1977 the Sixth Canadian Congress of Applied Mechanics, 1977.

[8] A.J. Chamkha, Unsteady flow of a dusty conducting fluid through a pipe, Mechanics Research Communications, Vol. 21, No. 3, P. 281-286, 1994.

[9] H.A. Attia, Unsteady flow of a dusty conducting non-Newtonian fluid through a pipe, Can. J. Phys., Vol. 81(3), (2003), P. 789-795.

[10] Nakayama, A., Koyama, H.: An analysis for friction and heat transfer characteristics of power-law non-Newtonian fluid flows past bodies of arbitrary geometrical configuration. Warme-und Stoffubertragung, Vol. 22, P. 29-37, 1988.

[11] Metzner, A.B.: Heat Transfer in non-Newtonian fluid. Adv. Heat Transfer, Vol. 2, P. 357-397, 1965.

[12] Sutton, G.W., Sherman, A.: Engineering Magnetohydrodynamics, McGrawHill, New York, 1965.

[13] Mitchell A.R., Griffiths, D.F.: The finite difference method in partial differential equations, John Wiley \& Sons, New York, 1980.

[14] Evans, G.A., Blackledge, J.M., Yardley, P.D.: Numerical methods for partial differential equations, Springer Verlag, New York, 2000. 


\section{Nomenclatures:}

$\rho \ldots$ the fluid-phase density

$\rho_{p} \ldots$ the particle-phase density

$\varphi \ldots$ the particle-phase volume fraction

$\sigma \ldots$ the fluid electrical conductivity

$\beta \ldots$ the Hall factor

... the apparent viscosity of the fluid

$p \ldots$ the particle-phase viscosity

$\tau_{0} \ldots$ the yield stress

$B_{o} \ldots$ the magnetic induction

$d$... circular pipe of radius

$K_{c} \ldots$ the coefficient of viscosity

$m \ldots$ the Hall parameter

$N \ldots$ a momentum transfer coefficient

$r \ldots$ the distance in the radial direction

$t$... time

$V \ldots$ the fluid-phase velocity

$V_{p} \ldots$ the particle-phase velocity 